\title{
LONG-TERM FOLLOW-UP OF 44 PATIENTS WITH ADENOCARCINOMA OF THE NASAL CAVITY AND SINUSES PRIMARILY TREATED WITH ENDOSCOPIC RESECTION FOLLOWED BY RADIOTHERAPY
}

\author{
Laura Van Gerven, MD, ${ }^{1}$ Mark Jorissen, MD, PhD, ${ }^{1,2}$ Sandra Nuyts, MD, PhD, ${ }^{2,3}$ \\ Robert Hermans, MD, PhD, ${ }^{2,4}$ Vincent Vander Poorten, MD, PhD ${ }^{1,2}$ \\ ${ }^{1}$ Department of Otorhinolaryngology-Head and Neck Surgery, University Hospitals Leuven, Leuven, Belgium. \\ E-mail: vincent.vanderpoorten@uzleuven.be \\ ${ }^{2}$ Leuvens Kanker Instituut (LKI), Leuven, Belgium \\ ${ }^{3}$ Department of Radiotherapy-Oncology, University Hospitals Leuven, Leuven, Belgium \\ ${ }^{4}$ Department of Radiology, University Hospitals Leuven, Leuven, Belgium
}

Accepted 17 June 2010

Published online in Wiley Online Library (wileyonlinelibrary.com). DOI: 10.1002/hed.21556

\begin{abstract}
Background. Endoscopic resection followed by radiotherapy as primary treatment for adenocarcinoma of the sinuses is emerging as an alternative to open resection.

Methods. A total of 44 patients primarily treated by an endoscopic approach followed by radiotherapy from 1992 to 2004 seen at our ENT-Department were analyzed for outcome and prognostic factors.

Results. Median follow-up was 61 months. Median followup of the patients alive at the end of the follow-up period was 100 months. For the 5-year follow-up, the overall survival, disease-specific survival, and recurrence-free survival were $63 \%$ $( \pm 7 \%$ SE), $82 \%( \pm 6)$, and $60 \%( \pm 8 \%)$, respectively. The overall survival, disease-specific survival, and recurrence-free survival after 100 months of follow-up were 53\% ( $\pm 8 \%), 72 \%$ $( \pm 9 \%)$, and $54 \%( \pm 9 \%)$, respectively. Four factors significantly influenced the disease-specific survival.

Conclusion. This study of a homogeneous cohort of patients with sinonasal adenocarcinoma treated by endoscopic resection and radiotherapy confirms that endoscopic resection
\end{abstract}

Correspondence to: V. Vander Poorten

(C) 2010 Wiley Periodicals, Inc. is a valid alternative to open resection. (C) 2010 Wiley Periodicals, Inc. Head Neck 00: 000-000, 2010

Keywords: adenocarcinoma; sinonasal tract; endoscopic sinus surgery; craniofacial resection; malignant tumors

The first reports on endoscopic surgery as an exclusive approach or in combination with an external approach for the treatment of malignant tumors of the nose and sinuses emerged in the late 1990s. ${ }^{1,2}$ Previous series of malignant tumors of the sinonasal tract treated with an endoscopic approach often included a diversity of histologic subtypes and treatment protocols. The cohorts were very small, due to the rarity of the pathology and the long natural history..$^{3-7}$ In Belgium, malignancies of the sinuses account for only $3 \%$ of the overall head and neck oncology diagnoses. ${ }^{8,9}$

In the last 2 years, studies with larger cohorts, more homogeneous histology, and treatment protocols were published reporting good 
local control of disease and survival after endoscopic resection. ${ }^{10,11}$ However, the relatively short follow-up and the difference in patient selection made it difficult to compare with the established gold standard of craniofacial resection (CFR) or lateral rhinotomy with medial maxillectomy followed by radiotherapy. ${ }^{12,13}$

\section{PATIENTS AND METHODS}

We reviewed a series of 44 patients with previously untreated adenocarcinoma of the ethmoid undergoing endoscopic resection and radiotherapy between 1992 and 2004 in our center. The results after 3 years of follow-up have already been published by Bogaerts et al. ${ }^{14}$ The same group of patients were now analyzed with longer follow-up periods. This homogeneous cohort of patients with sinonasal adenocarcinoma treated by endoscopic resection and postoperative radiotherapy has the longest follow-up period reported. In addition, we performed an analysis on the frequencies of the different sites of origin of this tumor and investigated more indepth the professional background of the patients in the cohort, to further document the earlier described etiologic role of occupational exposure to wood dust, as patients in our region often seemed to have worked in the flourishing wood industry in the region of our center. ${ }^{15-17}$ The correlation with the leather industry could not be analyzed due to the absence of this industry in our region.

Diagnostic Workup. The preoperative diagnostic workup consisted of a histopathological examination of an endoscopic biopsy specimen, with confirmation after surgery. Preoperative imaging included a dedicated multiplanar MRI study with injection of gadolinium for 40 patients (91\%), and all patients underwent a dedicated CT study with contrast agent injection. On the basis of this imaging, all tumors were staged according to the 2002 American Joint Committee on Cancer staging system. ${ }^{18}$ Routine blood tests, chest x-rays, and abdominal ultrasound scans were performed to evaluate the presence of regional and distant metastasis. Informed consent was obtained from all patients prior to any testing.

Selection Criteria for Endoscopic Resection. The status of all patients in the study were discussed during a multidisciplinary team meeting. Management options, including the possibility of requiring a CFR, were discussed along with factors related to the patients' general health and comorbidity. Preoperative imaging was crucial in the decision-making process.

The indications have been extended during the last years. Endoscopic resection was chosen when no invasion of dura, brain, or orbita was shown on preoperative CT or MRI scans, with the exception of some T4b lesions showing limited dural invasion that also were treated endoscopically.

Surgical Technique. An endoscopic resection of the tumor was performed with the patient under general anesthesia by 1 of the senior staff surgeons. For T1 tumors, the technique used can be described as an "en bloc" resection. After removal of the surrounding non-diseased structures for optimal approach, the small tumor was mobilized to allow a view of its insertion site and then removed in 1 piece. The majority of the tumors were mid-sized (T2) and were removed by a "phased" resection. This method consisted of a preparatory phase in which the tumor bulk was visualized. Using atraumatic suction, the insertion site of the tumor was defined as precisely as possible. The next step consisted of removing the tumor bulk using cold instruments and, lately, a microdebrider. In the last step, the tumor origin was broadly circumscribed by standard complete and radical frontospheno-ethmoidectomy, including a large middle meatal antrostomy and a resection of the upper third of the septum with a margin of about $1 \mathrm{~cm}$ where possible. For larger tumors (T3-T4), there was no option other than "piecemeal" removal, followed by an "en bloc" fronto-sphenoethmoidectomy and resection of the superior part of the nasal septum. If the tumor was unilateral, no contralateral radical surgery was undertaken. In cases of limited dural invasion, an endoscopic reconstruction was performed on the level of the roof of the ethmoid by a multilayer closure technique using fascia and mucosa.

Separate histological analyses were done on all mucosal resection borders and cribriform plate. Only in doubt, a frozen section biopsy control was performed.

Postoperative Radiotherapy. All patients had postoperative radiotherapy planned, generally starting within 5 weeks after surgery. Thirtyone patients received 3-dimensional (3D)-conformal radiotherapy applying a 3 -field photons 


\begin{tabular}{|c|c|c|c|}
\hline Prognostic factors & $\begin{array}{l}\text { Overall } \\
\text { survival }\end{array}$ & $\begin{array}{l}\text { Disease- } \\
\text { specific } \\
\text { survival }\end{array}$ & $\begin{array}{c}\text { Recurrence- } \\
\text { free } \\
\text { survival }\end{array}$ \\
\hline \multicolumn{4}{|l|}{ Clinical tumor characteristics } \\
\hline T classification & .468 & .947 & .292 \\
\hline T1-T2 vs T3-T4 & .156 & .953 & .978 \\
\hline \multicolumn{4}{|l|}{ Recurrence } \\
\hline First local recurrence & .973 & .025 & - \\
\hline Second local recurrence & .458 & .002 & $<.0001$ \\
\hline \multicolumn{4}{|l|}{ Development of metastasis } \\
\hline Regional & .074 & .003 & .001 \\
\hline Distant & .056 & $<.0001$ & $<.0001$ \\
\hline
\end{tabular}

Note: $\mathrm{p}$ values according to the log-rank test.

technique, using a once-daily fractionation scheme with a median dose of 60 Gy (range, 5070 Gy) in 30 fractions. Fields were defined by extension of the lesion and by aid of a planning CT scan. Eight patients received intensity modulated radiotherapy (IMRT). One patient refused radiotherapy, 1 patient's radiotherapy was discontinued due to intercurrent disease, and in another patient radiotherapy was stopped due to the development of lung metastases.

Statistical Analysis. The Statistical Package for Social Sciences (SPSS, version 15.0, Chicago, IL), commercially available, was used for statistical analysis. The overall survival, disease-specific survival, and recurrence-free survival rates were calculated by the Kaplan-Meier method. ${ }^{19}$

Univariate comparisons between groups were performed using the log-rank test. A value of $p \leq$ .05 was considered statistically significant.

All clinical data, surgical reports, preoperative and postoperative images, data on adjuvant therapy, and follow-up information were collected retrospectively. Variables evaluated for prognostic significance are reported in Table 1.

Follow-Up. Postoperative follow-up of the oncologic status consisted of clinical endoscopic examination of the nasal cavity every 2 months during the first year, every 4 months during the second year, every 6 months during the third year, and every 8,10 , and 12 months during the fourth, fifth, and sixth years, respectively, and then yearly thereafter. Additional imaging was only performed when a clinically suspicious lesion was endoscopically detected.

\section{RESULTS}

The study group included 44 patients, 43 men and 1 woman, with a mean age of 62 years at diagnosis. Presenting symptoms were unilateral rhinologic symptoms, with no predominant side.

The $\mathrm{T}$ classification, according to the 2002 American Joint Committee on Cancer staging system, is reported in Table 2.

All T4a tumors showed sphenoidal sinus involvement, whereas all T4b tumors showed erosion of the cribriform plate with dural invasion. Metastatic disease in the neck or at distant sites was not diagnosed at first diagnosis in any of the 44 patients.

All resection specimens were adenocarcinomas of the intestinal-type. At surgery and confirmed by histopathological examination, the tumor was found to involve the sites as shown in Figure 1. The olfactory cleft, as described in the study by Jankowski et $\mathrm{al}^{15}$ (middle and superior turbinate, posterior septum, and cribriform plate), was involved in $84 \%$ of all cases.

The site of recurrence most often included the posterior ethmoid cells $(21 \%)$, the ethmoid roof $(14 \%)$, and the posterior septum (11\%).

It is interesting to note that, except for 1 patient for whom no information was available, 42 of the remaining patients had an exposure to wood dust in their history.

Follow-up, ranging between 1 and 161 months (mean, 68 months; median, 61 months), was available for all patients. Follow-up of the patients alive at the end of the follow-up period ranged between 19 and 154 months (mean, 89 months; median, 100 months). Eleven patients died of causes unrelated to their tumors.

The 5-year overall survival was $63 \%( \pm 7 \%$ SE; Figure 2), with $72 \%( \pm 9 \%)$ for T2 tumors, $60 \%( \pm 22 \%)$ for T3 tumors, and $42 \%( \pm 14 \%)$ for $\mathrm{T} 4$ tumors.

The 1 patient with a $\mathrm{T} 1$ lesion is still alive after 8 years 5 months of follow-up. The overall survival after 100 months of follow-up was $53 \%$ $( \pm 8 \%)$.

\begin{tabular}{lc}
\hline \multicolumn{2}{c}{ Table 2. T classification. } \\
\hline T classification & No. of patients \\
\hline T1 & 1 \\
T2 & 26 \\
T3 & 5 \\
T4a & 9 \\
T4b & 3 \\
\hline
\end{tabular}




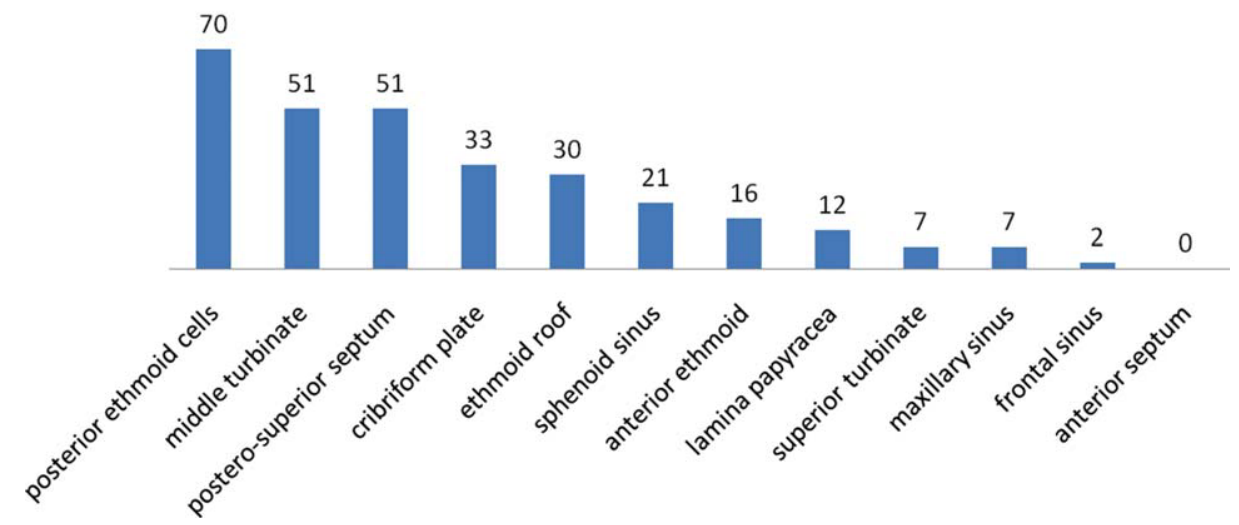

FIGURE 1. Subsites of tumor involvement percentages. [Color figure can be viewed in the online issue, which is available at wileyonlinelibrary.com.]

The 5-year disease-specific survival was $82 \%$ $( \pm 6 \%)$, with $83 \%( \pm 8 \%)$ for $\mathrm{T} 2$ tumors, $75 \%$ $( \pm 22 \%)$ for T3 tumors, and $80 \%( \pm 13 \%)$ for T4 tumors (Figure 3). The 100-month disease-specific survival was $72 \%( \pm 9 \%)$.

The mean recurrence-free interval, determined as the time between the day of surgery and the date of diagnosis of the first recurrence, was 27 months $( \pm 5)$. The 5-year recurrence-free survival was $60 \%( \pm 8 \%)$. The recurrence-free survival after 100 months of follow-up was $54 \%( \pm 9 \%)$. As in the study by Bogaerts et al, ${ }^{14}$ there was still no statistically significant influence of $\mathrm{T}$ classification on overall survival (log-rank $p=.468$ ), disease-specific survival (log-rank $p=.947)$, or on local control (log-rank $p=.292$ ).

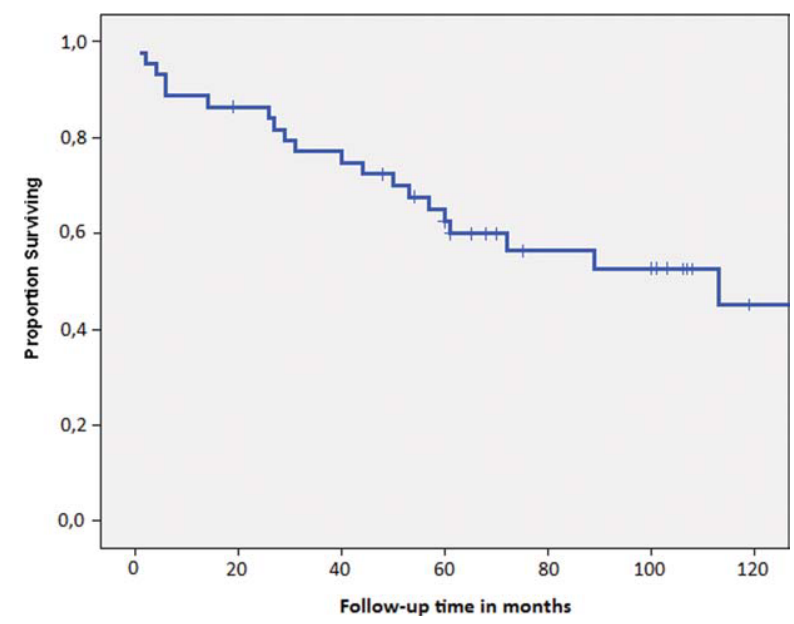

FIGURE 2. Overall survival of the whole group. [Color figure can be viewed in the online issue, which is available at wileyonlinelibrary.com.]
Sixteen of 44 patients (36\%) presented with a local recurrence. There was a statistically significant influence of recurrence on the disease-specific survival (log-rank $p=.025$; Figure 4).

The treatment protocols of the recurrences are reported in Table 3. Six of these remained free of tumor recurrence throughout the follow-up period. Ten patients presented with a second recurrence at a later date. There was a statistically significant influence of second recurrence on the disease-specific survival (log-rank $p=.002$ ) and the recurrence-free survival (log-rank $p=<.0001)$. Seven of the patients with a second recurrence were again treated by surgery, while the other 3 patients received supportive care only (Table 3). Distant

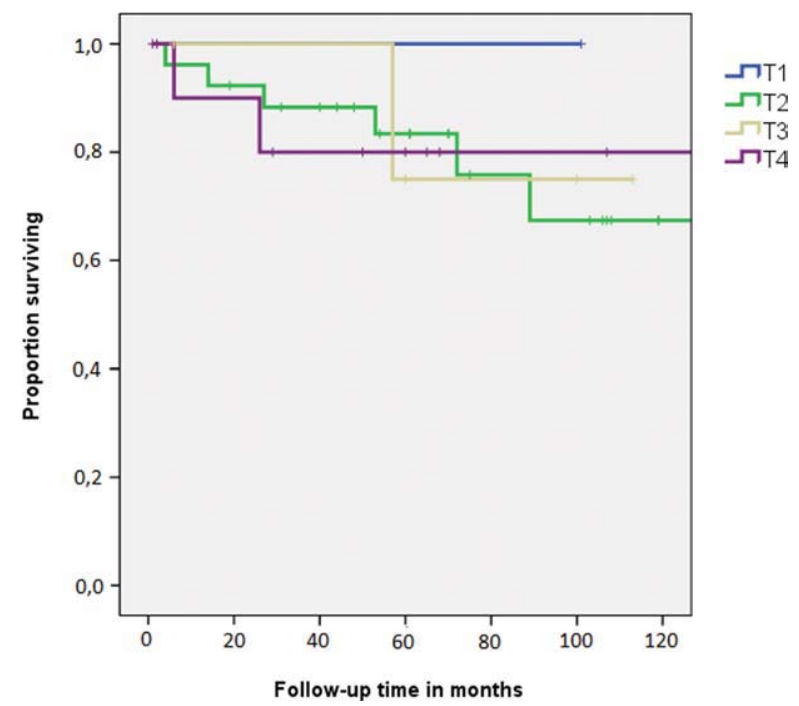

FIGURE 3. Disease-specific survival of the T-subgroups. [Color figure can be viewed in the online issue, which is available at wileyonlinelibrary.com.] 


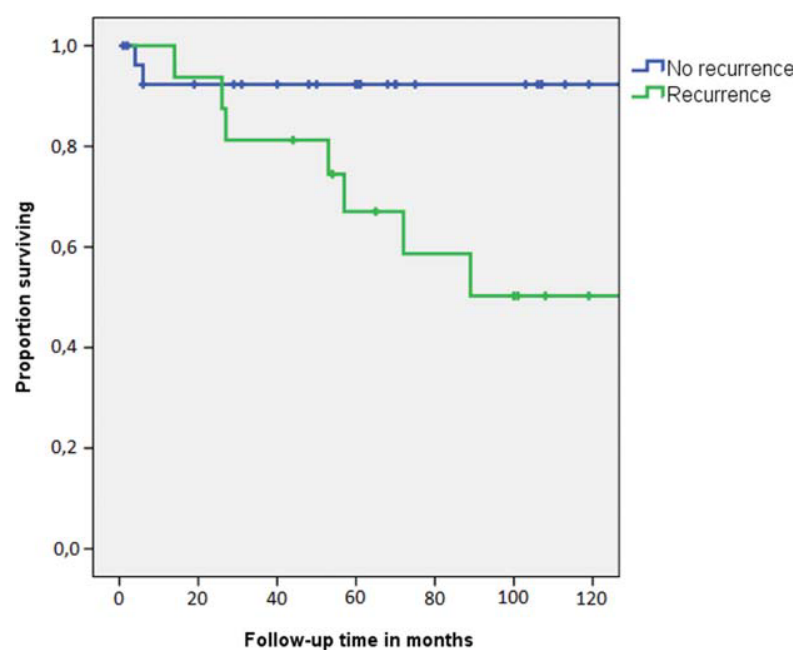

FIGURE 4. Disease-specific survival: recurrence versus no recurrence. [Color figure can be viewed in the online issue, which is available at wileyonlinelibrary.com.]

metastasis occurred in 7 patients, in 2 to the lungs, in 1 to the brain, and in 1 to the spine. Three patients had multiple metastases. Neck metastasis occurred in 2 patients who developed distant metastasis as well.

There was a statistically significant influence of the development of neck metastasis (log-rank $p=$ .003 ) and distant metastasis (log-rank $p=<.0001)$ on the disease-specific survival and recurrence-free survival (log-rank $p=.001$ and log-rank $p=<$ .0001 , respectively).

\section{DIsCussion}

Comparison to Open Resection. In evaluating outcome, endoscopic approaches should be compared with traditional external techniques: on 1 side with lateral rhinotomy for tumors not reaching the anterior skull base and on the other side with CFR for tumors in contact with or invading the anterior skull base. ${ }^{4,5}$ Because of the rarity of good studies on lateral rhinotomy, comparison is very difficult. In contrast, however, there are several reports of large patient cohorts treated with CFR with a long follow-up available in the literature. Howard et al, ${ }^{20}$ analyzing 308 patients treated with CFR with a follow-up of up to 25 years, showed a 5year disease-free survival of $59 \%$ for malignant tumors and $58 \%$ for adenocarcinomas specifically. In our series, the overall survival, diseasespecific survival, and recurrence-free percentages were $63 \%( \pm 7 \% \mathrm{SE}), 82 \%( \pm 6)$, and $60 \%$ $( \pm 8 \%)$, respectively. It is worth mentioning that an important part of our group of patients (39\%) had a T3 or T4a to T4b lesion.

In contrast with previous studies ${ }^{4,7,10}$ with relatively shorter follow-up time, the present study has sufficient follow-up to meaningfully compare the outcome of endoscopic resection to the established gold standard of CFR followed by radiotherapy. Equal survival and disease-specific survival curves are found for the 2 treatment protocols.

\begin{tabular}{|c|c|c|c|c|c|c|c|c|c|c|c|}
\hline $\begin{array}{c}\text { Date of } \\
\text { 1st surgery }\end{array}$ & $\begin{array}{c}\mathrm{T} \\
\text { classification }\end{array}$ & $\begin{array}{c}\text { Type } \\
\text { of surgery }\end{array}$ & $\mathrm{RT}$ & $\begin{array}{l}\text { Date } \\
1 \text { st R }\end{array}$ & $\begin{array}{l}\text { Treatment } \\
\text { 1st R }\end{array}$ & Date 2nd R & $\begin{array}{l}\text { Treatment } \\
\text { 2nd R }\end{array}$ & $\begin{array}{c}\text { Neck } \\
M\end{array}$ & $\begin{array}{l}\text { Distant } \\
\text { M }\end{array}$ & $\begin{array}{l}\text { FU } \\
\text { status }\end{array}$ & $\begin{array}{c}\text { Date of } \\
\text { death }\end{array}$ \\
\hline Jun/93 & T2 & ER R & 50 Gy & Aug/97 & ER R & I & I & $\mathrm{N}$ & $\mathrm{N}$ & DOC & Nov/06 \\
\hline Oct/94 & $\mathrm{T} 2$ & ER L & 50 Gy & Oct/95 & ER R & Dec/96 & SC & $\mathrm{N}$ & $\mathrm{N}$ & DOD & Mar/99 \\
\hline $\mathrm{Dec} / 95$ & $\mathrm{~T} 4 \mathrm{~b}$ & ER R & 60 Gy & Dec/96 & CFR & 1 & I & $\mathrm{N}$ & Lung & AWD & I \\
\hline Apr/98 & $\mathrm{T} 2$ & ER L & 60 Gy & Apr/04 & ER R & $\mathrm{Dec} / 04$ & ER L & $\mathrm{N}$ & $N$ & DOD & Sep/05 \\
\hline Oct/98 & $\mathrm{T} 2$ & ER R & 60 Gy & Apr/03 & ER R & Jun/08 & CFR & $N$ & $\mathrm{~N}$ & NED & 1 \\
\hline Jun/99 & $\mathrm{T} 2$ & ER R & 60 Gy & Nov/99 & $\mathrm{ER} R+\mathrm{CFR}$ & Apr/00 & SC & $\mathrm{N}$ & Multiple & DOD & $\mathrm{Jul} / 00$ \\
\hline Dec/99 & $\mathrm{T} 1$ & ER R & 60 Gy & Feb/01 & ER R & 1 & I & $\mathrm{N}$ & $N$ & NED & I \\
\hline Jun/00 & T3 & ER R & 60 Gy & Jan/03 & ER R & Aug/06 & CFR & $\mathrm{N}$ & Lung & AWD & l \\
\hline Jul/00 & $\mathrm{T} 2$ & ER R & $60 \mathrm{~Gy}$ & Sep/02 & $\mathrm{RS}$ & I & I & $\mathrm{N}$ & $N$ & DOC & Mar/04 \\
\hline Oct/99 & T2 & ER L & 60 Gy & Feb/01 & CFR & Sep/02 & ER R & $\mathrm{N}$ & $N$ & NED & 1 \\
\hline Oct/01 & $\mathrm{T} 4 \mathrm{~b}$ & ER L & 60 Gy & Dec/02 & CFR & Nov/03 & SC & $\mathrm{N}$ & Spine & DOD & $\mathrm{Dec} / 03$ \\
\hline Sep/02 & $\mathrm{T} 2$ & ER R & 70 Gy & May/07 & ER: debulking $(3 \times)$ & 1 & l & $\mathrm{N}$ & Brain & DOD & Sep/08 \\
\hline Feb/03 & T3 & $\mathrm{ER} L$ & 66 Gy & Apr/04 & ER L & Apr/06 & ER L & $P$ & Multiple & DOD & Nov/07 \\
\hline May/03 & $\mathrm{T} 4 \mathrm{a}$ & $E R R+E R L$ & 60 Gy & $\mathrm{Jan} / 04$ & ER & Mar/05 & ER & $\mathrm{N}$ & $\mathrm{N}$ & NED & i \\
\hline Mar/04 & $\mathrm{T} 2$ & ER R & 56 Gy & Mar/08 & ER R & Sep/08 & ER R & $\mathrm{N}$ & N & NED & I \\
\hline Jul/04 & $\mathrm{T} 2$ & ER L & 60 Gy & Apr/05 & MRND L & 1 & I & $\mathrm{P}$ & Multiple & DOD & Nov/06 \\
\hline
\end{tabular}

Abbreviations: $R T$, radiotherapy; $R$, recurrence: $M$, metastases; $F U$, follow-up: $E R R$, endoscopic surgery at the right side; Gy, Gray: $N$, negative: DOC death other cause: ER L, endoscopic surgery at the left side: SC, supportive care: DOD, death of disease: CFR, craniofacial resection: AWD, alive with disease; NED, no evidence of disease; RS, resection of septum + ossa nasalia and skin with reconstruction; $P$, positive; MEND L, modified radical neck dissection (left side). 


\begin{tabular}{|c|c|c|c|c|c|c|c|}
\hline Study & No. of patients & $\%$ Adenoca & 5 -y OS & 5-y DSS & 5-y RFS & Mean Fu, mo & Treatment \\
\hline Howard $2006^{20}$ & 259 & $20 \%$ & $65 \%$ & $59 \%$ & - & 63 & CFR \\
\hline Lund $2007^{4}$ & 49 & $31 \%$ & $83 \%$ & - & $72 \%$ & 36 & $\mathrm{EEA} \pm \mathrm{RT}$ \\
\hline Nicolai $2007^{7}$ & 16 & $75 \%$ & - & $93 \%$ & $87 \%$ & 47 & $\mathrm{EEA} \pm \mathrm{RT}$ \\
\hline Choussy $2008^{11}$ & 478 & $100 \%$ & $64 \%$ & - & - & 58.7 & $1.5 \% \mathrm{EEA} \pm \mathrm{RT}$ \\
\hline Nicolai $2008^{10}$ & 184 & $37 \%$ & - & $94 \%$ & - & 34 & EEA $(73 \%)$ or CEA $(27 \%) \pm R T$ \\
\hline Bogaerts $2008^{14}$ & 44 & 100 & 83 & 83 & 62 & 42 & $\mathrm{EEA}+\mathrm{RT}$ \\
\hline Hanna $2009^{24}$ & 120 & 14 & 76 & 87 & 85 & 37 & $\mathrm{EEA}(77.5 \%)$ or $\mathrm{CEA}(22.5 \%) \pm \mathrm{RT}$ \\
\hline This study & 44 & 100 & 63 & 82 & 60 & 68 & $\mathrm{EEA}+\mathrm{RT}$ \\
\hline
\end{tabular}

Abbreviations: OS, overall survival; DSS, disease-specific survival; RFS, recurrence-free survival; FU, follow-up time in months; CFR, craniofacial resection; EEA, exclusive endoscopic approach; RT, radiotherapy; CEA, cranio-endoscopic approach.

Besides comparable survival curves for both surgical techniques, other factors can play an important role to promote endoscopic resection as a promising alternative technique to open surgery. Factors that favor the use of endoscopic resection are avoidance of facial incisions and osteotomies, short hospitalization, and a very low morbidity. ${ }^{21}$ Even in the hands of experts, CFR is still associated with rates of mortality (4.7\%) and morbidity (36.3\%) that must be considered, as published by Ganly et al. ${ }^{22}$

Endoscopic resections are associated with a lower rate of complications even in cases requiring dura resection (radiological T4b in our series). In fact, overall we observed no major complications associated with endoscopic resection.

Although there are a lot of advantages to treat an adenocarcinoma of the sinuses with an endoscopic resection, there are limitations as well. There is the debate about obtaining clear margins when resecting a tumor and subsequently about the limits of an endoscopic approach. Generally, we can state that anatomy dictates where adequate margins can be obtained, especially toward the anterior skull base.

Piecemeal resection as an alternative to "en bloc" resection seems contradictory to the cornerstone of oncological surgery that focuses on a maximal in 1 piece resection, avoiding the risk of tumor spilling. ${ }^{23}$ But no data supporting this hypothesis in malignant paranasal sinus disease were published, and en bloc resection using external approaches is also frequently impossible.

Comparison to Other Studies using Endoscopic Resection. There is a general consensus in the literature that more homogeneity in histology and treatment protocols is necessary to compare the survival curves in a meaningful way. To date, Bogaerts et al $^{14}$ had the most homogeneous group. Another problem is the small patient cohorts included in the studies making it difficult to identify statistically significant factors influencing oncological outcome. However, the recent study of Nicolai et $\mathrm{al}^{10}$ and Choussy et $\mathrm{al}^{11}$ are exceptions of this remark (Table 4).

Comparing our findings with those of Nicolai et $\mathrm{al}^{7,10}$ and Lund et $\mathrm{al}^{4}{ }^{4}$ it is remarkable that they have more intense postoperative follow-up schemes by imaging and endoscopic examinations. Nicolai et $\mathrm{al}^{7,10}$ has smaller intervals for detection of recurrence: 10 months and 15 months, respectively, in their last 2 studies, in comparison with 25 months in our previous study. ${ }^{14}$ Their more intense follow-up schemes with imaging can explain their smaller intervals for detection of recurrence. However, this did not change the percentage of recurrences because long-term follow-up was available.

The study by Lund et $\mathrm{al}^{4}$ did not report on their recurrence-free interval.

In our study, local recurrences are more frequent than distant metastases, and the local recurrences are responsible for the majority of deaths.

Postoperative Radiotherapy. The limitations of surgery alone are obvious given the frequent presentation of advanced disease. Postoperative radiotherapy improves local control regardless of surgical margins. ${ }^{25}$ At our center, patients with sinonasal cancer have been treated for many decades with the combination of surgery and radiotherapy $(\mathrm{RT})$.

Since 2003, IMRT has been routinely used at our center for the treatment of sinonasal cancer. IMRT allows sparing of normal tissues in contrast with conventional or even 3D-conformal $\mathrm{RT}$ which is associated with, especially in these cases, visual toxicity. ${ }^{26}$ Recently, several studies confirmed that besides the low risk of complications with IMRT, IMRT has similar local control 


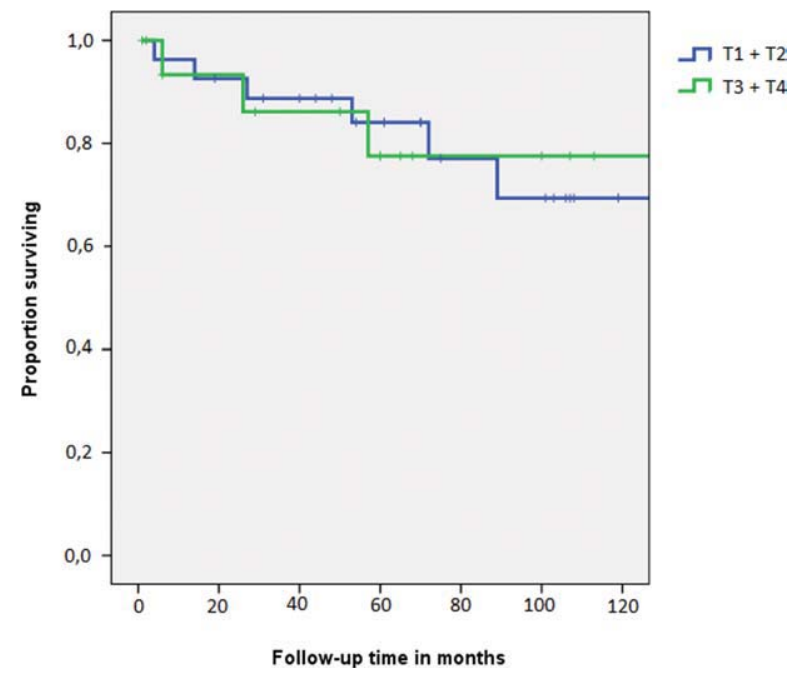

FIGURE 5. Disease-specific survival: $\mathrm{T} 1+\mathrm{T} 2$ versus $\mathrm{T} 3+\mathrm{T} 4$. [Color figure can be viewed in the online issue, which is available at wileyonlinelibrary.com.]

and survival rates as conventional RT or $3 \mathrm{D}-$ conformal RT techniques. ${ }^{27,28}$

Prognostic Factors in Ethmoid Adenocarcinomas. As in the study by Bogaerts et al, ${ }^{14}$ there was still no statistically significant influence of $\mathrm{T}$ classification on overall survival, disease-specific survival, or recurrence-free survival. Even when the small tumors (T1-T2) and the T3 to T4 lesions were grouped, no influence (Table 1) could be proven. This could be due to lack of statistical power (Figure 5).

Choussy et $\mathrm{al}^{11}$ published a study about patients with exposure to wood or leather who were found to have a better prognosis. In this series, we had only 1 patient without exposure to wood dust, therefore, we could not verify this factor.

There was only 1 study $^{11}$ capable of finding factors significantly associated with the survival rate of adenocarcinomas of the sinuses. Given the large study population of 418 patients, statistical power to find these factors was sufficient. Factors influencing survival were predominantly the size of the lesion (T4), extension to the lymph nodes, and intracranial involvement. Surprisingly, development of distant metastasis could not be shown to have a statistically significant impact on survival.

In our current series, 7 patients developed metastasis, which significantly influenced the disease-specific survival $(p=<.0001)$, and recurrence-free survival $(p=<.0001)$, a first and a second recurrence also influenced the diseasespecific survival $(p=.025$ and $p=.002$, respec- tively), although this was not surprising, this had not been described before. We could confirm that extension to the lymph nodes significantly influenced the disease-specific survival $(p=<.0001)$ and the recurrence-free survival $(p=<.0001)$.

\section{CONCLUSION}

In this homogeneous cohort of patients with ethmoidal adenocarcinoma treated by endoscopic resection and postoperative radiotherapy and with a solid follow-up of 100 months, the 5-year disease-specific survival of $82 \%( \pm 6 \%)$ confirms that this approach, when properly planned and performed by experienced surgeons, is an effective alternative to open approaches.

\section{REFERENCES}

1. Stammberger H, Anderhuber W, Walch C, Papaefthymiou G. Possibilities and limitations of endoscopic management of nasal and paranasal sinus malignancies. Acta Otorhinolaryngol Belg 1999;53:199-205.

2. Goffart Y, Jorissen M, Daele J, et al. Minimally invasive endoscopic management of malignant sinonasal tumours. Acta Otorhinolaryngol Belg 2000;54:221-232.

3. Poetker DM, Toohill RJ, Loehrl TA, Smith TL. Endoscopic management of sinonasal tumors: a preliminary report. Am J Rhinol 2005;19:307-315.

4. Lund V, Howard DJ, Wei WI. Endoscopic resection of malignant tumors of the nose and sinuses. Am J Rhinol 2007;21:89-94.

5. Batra PS, Citardi MJ, Worley S, Lee J, Lanza DC. Resection of anterior skull base tumors: comparison of combined traditional and endoscopic techniques. Am J Rhinol 2005;19:521-528.

6. Podboj J, Smid L. Endoscopic surgery with curative intent for malignant tumors of the nose and paranasal sinuses. Eur J Surg Oncol 2007;33:1081-1086.

7. Nicolai P, Castelnuovo P, Lombardi D, et al. Role of endoscopic surgery in the management of selected malignant epithelial neoplasms of the naso-ethmoidal complex. Head Neck 2007;29:1075-1082.

8. Mosesson RE, Som PM. The radiographic evaluation of sinonasal tumors: an overview. Otolaryngol Clin North Am 1995;28:1097-1115.

9. Shah JP, Kraus DH, Bilsky MH, Gutin PH, Harrison LH, Strong EW. Craniofacial resection for malignant tumors involving the anterior skull base. Arch Otolaryngol Head Neck Surg 1997;123:1312-1317.

10. Nicolai P, Battaglia P, Bignami M, et al. Endoscopic surgery for malignant tumors of the sinonasal tract and adjacent skull base: a 10-year experience. Am J Rhinol 2008;22:308-316.

11. Choussy O, Ferron C, Védrine PO, et al. Adenocarcinoma of Ethmoid: a GETTEC retrospective multicenter study of 418 cases. Laryngoscope 2008;118:437-443.

12. McCutcheon IE, Blacklock JB, Weber RS, et al. Anterior transcranial (craniofacial) resection of tumors of the paranasal sinuses: surgical technique and results. Neurosurgery 1996;38:471-479; discussion 479-480.

13. Patel SG, Singh B, Polluri A, et al. Craniofacial surgery for malignant skull base tumors: report of an international collaborative study. Cancer 2003;98:1179-1187. 
14. Bogaerts S, Vander Poorten V, Nuyts S, Van den Bogaert W, Jorissen M. Results of endoscopic resection followed by radiotherapy for primarily diagnosed adenocarcinomas of the paranasal sinuses. Head Neck 2008;30:728-736.

15. Jankowski R, Georgel T, Vignaud JM, et al. Endoscopic surgery reveals that woodworkers' adenocarcinomas originate in the olfactory cleft. Rhinology 2007;45:308314.

16. Bimbi G, Saraceno MS, Riccio S, Gatta G, Licitra L, Cantù G. Adenocarcinoma of ethmoid sinus: an occupational disease. Acta Otorhinolaryngol Ital 2004;24:199-203.

17. Moreau JJ, Bessede JP, Heurtebise F, et al. Adenocarcinoma of the ethmoid sinus in woodworkers. Retrospective study of 25 cases. [Article in French] eurochirurgie 1997;43:111-117.

18. Sobin LH, Wittekind C. TNM classification of malignant tumours. 5th edition. New York: Wiley-Liss; 2002.

19. Kaplan EL, Meier P. Nonparametric estimation from incomplete observations. J Am Stat Assoc 1958;53:457481.

20. Howard DJ, Lund VJ, Wei WI. Craniofacial resection for tumors of the nasal cavity and paranasal sinuses: a 25year experience. Head Neck 2006;28:867-873.

21. Cohen MA, Liang J, Cohen IJ, Grady MS, O'Malley BW Jr, Newman JG. Endoscopic resection of advanced anterior skull base lesions: Oncologically safe? ORL J Otorhinolaryngol Relat Spec 2009;71:123-128.
22. Ganly I, Patel SG, Singh B, et al. Complications of craniofacial resection for malignant tumors of the skull base: report of an International Collaborative Study. Head Neck 2005;27:445-451.

23. Dave SP, Bared A, Casiano RR. Surgical outcomes and safety of transnasal endoscopic resection for anterior skull tumors. Otolaryngol Head Neck Surg 2007;136: 920-927.

24. Hanna E, DeMonte F, Ibrahim S, Roberts D, Levine N, Kupferman M. Endoscopic resection of sinonasal cancers with and without craniotomy: oncologic results. Arch Otolaryngol Head Neck Surg 2009;135:1219-1224.

25. Blanco AI, Chao KS, Ozyigit G, et al. Carcinoma of paranasal sinuses: long-term outcomes with radiotherapy. Int J Radiat Oncol Biol Phys 2004;59:51-58.

26. Takeda A, Shigematsu N, Suzuki S, et al. Late retinal complications of radiation therapy for nasal and paranasal malignancies: relationship between irradiated-dose area and severity. Int $J$ Radiat Oncol Biol Phys 1999;44:599-605.

27. Dirix P, Nuyts S, Vanstraelen B, et al. Post-operative intensity-modulated radiotherapy for malignancies of the nasal cavity and paranasal sinuses. Radiother Oncol 2007;85:385-391.

28. Dirix P, Nuyts S, Geussens Y, et al. Malignancies of the nasal cavity and paranasal sinuses: long-term outcome with conventional or three-dimensional conformal radiotherapy. Int J Radiat Oncol Biol Phys 2007;69:1042-1050. 